\title{
ALTERAÇÕES OSTEOMUSCULARES EM TÉCNICOS DE ENFERMAGEM EM UM AMBIENTE HOSPITALAR
}

\author{
Caroline Souza Andrade Rocha Fisioterapeuta, graduada pela Faculdade \\ Social da Bahia. \\ Carolina Barbosa da Silva Fisioterapeuta, graduada pela Faculdade \\ Social da Bahia.
Mansueto Gomes Neto Fisioterapeuta, mestre em Ciências da Reabilitação pela UFMG. Docente da Universidade Federal da Bahia. \\ Bruno Prata Martinez Fisioterapeuta do Hospital Aliança. Docente
}

\begin{abstract}
Resumo
Introdução: As afecções osteomusculares relacionadas ao trabalho são consideradas um problema de saúde pública. Surgem de forma insidiosa e podem progredir, causando incapacidade laboral temporária ou permanente. A equipe de técnicos de enfermagem no ambiente hospitalar é uma população de risco para alterações osteomusculares, tornando-se necessária a avaliação da prevalência destes sintomas. Objetivo: Identificar alterações osteomusculares em técnicos de enfermagem em um ambiente hospitalar. Materiais e métodos: Trata-se de um estudo descritivo e transversal, realizado com 60 técnicos de enfermagemde um ambiente hospitalar, com uma idade média de 32,8 $\pm 8,9$ anos, e com predomínio do sexo feminino (93,3\%).Foram coletados os dados demográficos como idade, altura, peso, gênero, estado civil, local de trabalho, tempo de serviço e jornada de trabalho. O instrumento de avaliação utilizado para identificar a ocorrência de alterações osteomusculares foi o Questionário Nórdico de Sintomas Osteomusculares (QNSO).Resultados: A prevalência de dor em algumas regiões do corpo nos últimos 12 meses foi de 63,3\% na região lombar, $56,7 \%$ em tornozelos e pés e $51,7 \%$ em ombros. Nos últimos 7 dias $38,3 \%$ dos indivíduos sentiram dor nos tornozelos e pés e 16,7\% faltaram ao trabalho por conta de dor nos joelhos. Os participantes tinha uma média de 4,3 $\pm 5,9$ anos de atuação no serviço.Conclusão: Foi observado uma alta prevalência de sintomas osteomusculares em técnicos de enfermagem, principalmente na região lombar, nos tornozelos, pés e ombros,provavelmente devido a posturas inadequadas e a alta demanda de trabalho.
\end{abstract}

Palavras-chave:Técnicos de enfermagem;Sintomas osteomusculares; Hospital.

\section{MUSCULOSKELETAL CHANGES IN NURSING TECHNICALIN AHOSPITAL ENVIRONMENT}

\begin{abstract}
Introduction: The Work-related Musculoskeletal Disorders (WMSD) is considered a public health problem. They appear in a insidious way and can progress, causing temporary or permanent labor disability. The staff of nurse technicians in a hospital environment it's a risk population for musculoskeletal disorders, which makes necessary to evaluate the prevalence of these symptoms. Purpose: Identify musculoskeletal disorders in a nurse staff on a hospital environment. Materials and methods: It is a descriptive and transversal study performed in 60 nurse technicians on a hospital environment, with an average age from 32,8 \pm 8,9 years-old, predominant female gender (93.3\%). There were collected demographic data such as age, height, weight, gender, marital status, workplace, time of service and workday (shift). The assessment tool used to identify the occurrence of musculoskeletal changes was the Nordic Musculoskeletal Questionnaire (NMQ). Results: The prevalence of pain in some body regions in the last 12 months was $63,3 \%$ on the lower back, $56.7 \%$ on ankles and feet, and $51,7 \%$ in the shoulder region. In the last 7 days $38,3 \%$ of the individuals experienced pain on the ankles and feet and $16,7 \%$ missed work do to pain in the knees. The participants had an average of 4,3 $\pm 5,9$ years of work in the field. Conclusion: It was observed a high prevalence of musculoskeletal symptoms in nurse technicians, especially in the low back, ankles, feet and shoulders, probably because of the inappropriate posture and high request of work.
\end{abstract}

Keywords: Nursing technical;Cumulative trauma disorders; Hospital. 


\section{INTRODUÇÃO}

A doença osteomuscular relacionadas ao trabalho (LER/DORT) é considerada como um problema de saúde pública, ${ }^{(1)}$ e está situada entre as mais frequentes doenças do trabalho no mundo industrializado, ${ }^{(2)}$ surgindo de forma insidiosa e com uma progressão que pode causar incapacidade laboral temporária ou permanente. ${ }^{(3,4)}$ A necessidade do destaque profissional para promoção de cargo é um dos fatores principais que leva ao trabalhador a uma maior exigência da sua tarefa, gerando consequiências na sua saúde e qualidade de vida. $^{(5,6)}$

Os dados disponíveis pelo Instituto de Seguridade Social (INSS) demonstram o alto índice de LER/DORT, provocando umas das maiores causas de afastamento. ${ }^{(7)}$ Trabalhadores acometidos buscam ajuda pela Previdência Social através do auxílio-acidente e aposentadoria por invalidez, gerando aumento dos custos para o governo. ${ }^{(5,8)}$

A descoberta do diagnóstico provoca no indivíduo um sentimento de impotência, causando angústia e depressão. ${ }^{(9,10)}$ Dessa forma, a contínua procura por tratamentos torna-se incessante, e em alguns casos pode levar o profissional a longos períodos de afastamento do trabalho. ${ }^{(11,12)}$ Muitos trabalhadores sofrem com dores musculares devido a posturas inadequadas e aos grandes esforços realizados no ambiente de trabalho, ${ }^{(13)}$ sendo importante destacar que estas doenças laborais não estão associadas apenas a sintomas físicos, estando relacionadas também a organização do trabalho, relacionamentos interpessoais como também aos fatores ergonômicos. ${ }^{(11,13,14)}$

Determinados tipos de atividades apresentam maior probabilidade de lesões, gerando um grupo de risco para LER/DORT dentro das empresas. A equipe de enfermagem é um exemplo de grupo que está constantemente exposto a diversos riscos de saúde, principalmente durante a manipulação dos pacientes. ${ }^{(15)}$ Uma excessiva carga de trabalho dos técnicos de enfermagem pode ser um fator de risco, podendo colocar a vida dos pacientes em perigo, por conta do baixo rendimento e falta de atenção durante o serviço realizado. ${ }^{(16,17)}$

Dentre os distúrbios mais frequentes na equipe de enfermagem, destacam-se as lombalgias que são decorrentes de traumas cumulativos. ${ }^{(14,15)}$ Um fator agravante para a recorrência dos sintomas em técnicos é o retorno para o serviço apresentando os mesmos sintomas que gerou o seu afastamento do trabalho. ${ }^{(18)}$ Sabendo-se da atividade laboral de risco nestes profissionais, torna-se importante a descrição da freqüência de alterações 
osteomusculares em técnicos de enfermagem em um ambiente hospitalar, para uma possível realização de medidas preventivas.

\section{MATERIAIS E MÉTODOS}

Trata-se de um estudo transversal do tipo descritivo e quantitativo, realizado em 2010 no hospital Santo Antônio-Obras Sociais Irmã Dulce, na cidade de Salvador, Bahia. A pesquisa foi aprovada pelo Comitê de Ética do hospital, com protocolo 36/10. O mesmo foi realizado com o intuito de identificar lesões osteomusculares em técnicos de enfermagem por conta da sobrecarga de trabalho. Foram incluídos 60 técnicos de enfermagem, de ambos os sexos e que atuassem na unidade de terapia intensiva e enfermaria adulta. Foi utilizado como critério de exclusão pessoas que atuassem em outra profissão que não a de técnico de enfermagem ou que estivesse afastado por causa da LER/DORT.

Todos os participantes foram esclarecidos quanto aos procedimentos e objetivos do estudo e os mesmos assinaram um termo de consentimento livre e esclarecido baseado na resolução 196/96. ${ }^{(19)}$ Foram coletados os dados demográficos como: nome, idade, altura, peso, gênero, estado civil, local de trabalho, tempo de serviço e jornada de trabalho. Em seguida foi aplicado instrumento de avaliação denominado Questionário Nórdico de Sintomas Osteomusculares (QNSO), ${ }^{(20,21)}$ o qual é utilizado para identificar a ocorrência de alterações osteomusculares geradas pelo trabalho.

Para análise dos dados demográficos e clínicos, foram utilizadas estatísticas descritivas. Os dados de variáveis contínuas foram avaliados com medidas de tendência central e dispersão e expressos como médias, medianas e desvio-padrão. Os dados de variáveis dicotômicas ou categóricas foram avaliados com medidas de freqüência e expressos como porcentagens, sendo que para análise da associação entre a presença de sintomas no questionário Nórdico e o tempo de serviço e a carga horária semanal de trabalho, foi utilizado o teste Qui-quadrado. O tempo de serviço foi estratificado em dois grupos, sendo um com menos que 3 anos e o outro com mais de 3 anos de atuação. Já a carga horaria semanal foi dividida em com mais de 36 horas e com menos de 36 horas por semana. O nível de significância utilizado foi menor que $5 \%(0,05)$. com o uso do software SPSS (StatisticalPackage for theSocialSciences) for Windows (versão 14.0). Esses dados foram apresentados com a elaboração de tabelas e descritos. 


\section{RESULTADOS}

No presente estudo foram selecionados 60 técnicos de enfermagem com uma média de idade de 32,8 \pm 8,9 anos, existindo predomínio do sexo feminino (93,3\%). Quanto ao estado civil, verificou-se que 53,3\% eram solteiros e a média do índice de massa corpórea (IMC) foi de $25,6 \pm 4,7 \mathrm{~kg} / \mathrm{m}^{2}$.

Analisando a variável tempo de atuação no serviço, foi encontrada uma média de 4,3 $\pm 5,9$ anos, com uma média da jornada semanal de trabalho de 4,9 $\pm 1,4$ dias e da jornada diária de trabalho 9,5 $\pm 4,1$ horas.

Tabela 1 - Características sóciodemográficas e de trabalho dos profissionais ( $\mathrm{n}=60$ ) no hospital de Salvador, 2010

\begin{tabular}{|c|c|c|c|}
\hline Caracterísitca & Categoria & $\mathrm{n}$ & $\%$ \\
\hline Idade & & $32,8 \pm 8,9$ & \\
\hline \multirow[t]{2}{*}{ Sexo } & Masculino & 4 & 6,7 \\
\hline & Feminino & 56 & 93,3 \\
\hline $\begin{array}{l}\text { Tempo de atuação } \\
\text { profissional (anos) }\end{array}$ & & $4,9 \pm 1,4$ & \\
\hline Carga horária (dia) & & $9,5 \pm 4,1$ & \\
\hline IMC $\left(\mathrm{kg} / \mathrm{m}^{2}\right)$ & & $25,6 \pm 4,7$ & \\
\hline \multirow{2}{*}{ Estado Civil } & Solteiro & 32 & $53,3 \%$ \\
\hline & Casado & 28 & $46,7 \%$ \\
\hline
\end{tabular}

Em relação ao local de trabalho, $70 \%$ da população pesquisada trabalhavam apenas no referido hospital, $10 \%$ atuavam no hospital e em atendimento domiciliar e $20 \%$ trabalhavam em mais de um hospital. Referente ao local de trabalho dentro no hospital, 91,6\% dos participantes trabalhavam em enfermaria adulto e 8,3\% na UTI adulto.

Em relação aos problemas como dor, formigamento ou dormência ocorrido nos últimos 12 meses que antecederam a pesquisa, a maior incidência de queixas foi observada na coluna lombar, seguido de tornozelos, pés e ombros, vide descrição na Tabela 2. 
Tabela 2 - Nos últimos 12 meses, você teve problemas (como dor, formigamento/dormência,) em:

\begin{tabular}{ccc}
\hline Região Anatômica & SIM & $(\%)$ \\
\hline Pescoço & 22 & $36,70 \%$ \\
Ombros & 31 & $51,70 \%$ \\
Costas & 28 & $46,70 \%$ \\
Cotovelos & 4 & $6,70 \%$ \\
Punhos/Mãos & 19 & $31,70 \%$ \\
Lombar & 38 & $63,30 \%$ \\
Quadril/Coxas & 21 & $35,00 \%$ \\
Joelhos & 26 & $43,30 \%$ \\
Tornozelos/Pés & 34 & $56,70 \%$ \\
\hline
\end{tabular}

A segunda pergunta do QNOS se referia ao absenteísmo por conta das dores em alguma região corporal nos últimos 12 meses. Nessa questão os resultados foram pouco relevantes, sendo a maior porcentagem para a região dos joelhos (16,7\%), em segundo lugar ficou a região lombar, com 11,7\%, e em terceiro lugar, duas regiões referidas no questionário apresentaram a mesma porcentagem, com 8,3\% para as regiões dos punhos/mãos e tornozelos/pés. Poucos funcionários deixaram de trabalhar ou de realizar seus afazeres domésticos e atividades de lazer, por conta da dor.

De acordo com os resultados encontrados na Tabela 3, os participantes da pesquisa também procuram por profissionais de saúde, devido aos sintomas apresentados. O que mais levou o trabalhador a faltar o trabalho e a procurar por esses profissionais foi dor na região dos joelhos, seguida da região lombar, embora os joelhos não tenham sido o local mais acometido pelas alterações osteomusculares, como pôde ser observado na tabela anterior. Apesar dos joelhos não terem sido a região de maior queixa álgica, esta foi a região que mais levou os trabalhadores a procurarem os profissionais da área de saúde para sanarem suas dores. 
Tabela 3 - Nos últimos 12 meses, fez consulta com algum profissional da área de saúde (médico, fisioterapeuta) por causa dessa condição em:

\begin{tabular}{ccc}
\hline Região Anatômica & SIM & $(\%)$ \\
\hline Pescoço & 6 & $10,00 \%$ \\
Ombros & 7 & $11,70 \%$ \\
Costas & 6 & $10,00 \%$ \\
Cotovelos & 2 & $3,30 \%$ \\
Punhos/Mãos & 5 & $8,30 \%$ \\
Lombar & 12 & $20,00 \%$ \\
Quadril/Coxas & 7 & $11,70 \%$ \\
Joelhos & 13 & $21,70 \%$ \\
Tornozelos/Pés & 12 & $20,00 \%$ \\
\hline
\end{tabular}

Com relação aos dados obtidos referentes à ocorrência de alterações osteomusculares nos últimos 7 dias, houve uma prevalência para essas dores nas regiões dos tornozelos/pés e coluna lombar, sendo observado na Tabela 4. Estes resultados foram proporcionalmente similares aos encontrados nas duas questões anteriores do QNSO, sendo demonstrado o absenteísmo e a ocorrência de dores em algumas regiões corporais. 
Tabela 4 - Nos últimos 7 dias você teve algum problema em:

\begin{tabular}{ccc}
\hline Região Anatômica & SIM & $(\%)$ \\
\hline Pescoço & 16 & $26,70 \%$ \\
Ombros & 19 & $31,70 \%$ \\
Costas & 15 & $25,00 \%$ \\
Cotovelos & 2 & $3,30 \%$ \\
Punhos/Mãos & 9 & $15,00 \%$ \\
Lombar & 22 & $36,70 \%$ \\
Quadril/Coxas & 15 & $25,00 \%$ \\
Joelhos & 18 & $30,00 \%$ \\
Tornozelos/Pés & 23 & $38,30 \%$ \\
\hline
\end{tabular}

Ao avaliar a comparação entre as perguntas do questionário Nórdico nas diversas regiões, com o tempo de atuação profissional (menor e maior que 3 anos) e com a carga horária semanal (menor e maior que 36 horas), houve apenas significância $(p=0,02)$ na questão 4 na região lombar, sendo que a dor nos últimos 7 dias foi maior no grupo com maior carga horaria semanal.A média de idade foi maior no grupo com tempo de atuação maior que 3 anos (38,5 \pm 9,5 anos) em relação aos com tempo menor que 3 anos $(29,11 \pm 6,2$ anos). 


\section{DISCUSSÃO}

O presente estudo observou uma alta prevalência de sintomas osteomusculares em técnicos de enfermagem no referido hospital, destacando-se a região lombar, tornozelos e ombros, nos últimos doze meses, convergindo com o estudo realizado por Carvalho e Alexandre em 2006.

Mergener, Kehrig eTraebert ${ }^{(6)}$ encontrou uma alta prevalência de sintomas músculoesqueléticos $(72,8 \%)$ em bancários, sendo que a principal região acometida foi a do trapézio $(31,2 \%)$. Já na pesquisa em questão, a maior prevalência de sintomas ocorreu na região lombar $(63,3 \%)$. Esses estudos comprovam que, independente da função laboral exercida, a carga excessiva de trabalho, gera compensações e dores musculares de forma crônica.

Em outro estudo com uma amostra de 502 bancários, pôde-se constatar a prevalência de distúrbios musculares e seus fatores associados. Da população em estudo, $60 \%$ relatou dor músculoesquelética no último ano e $19 \%$ tiveram que evitar o trabalho por causa das dores. ${ }^{(22)}$ $\mathrm{Na}$ atual pesquisa também foi possível verificar a ausência ao trabalho, devido às alterações osteomusculares, sendo que $91,6 \%$ dos participantes relataram ter sentido dor nos últimos 12 meses, e $26,6 \%$ se ausentaram do trabalho por conta dos sintomas. De forma comparativa, nota-se que em ambos os estudos houveram baixos percentuais relacionados ao absenteísmo, por conta das dores geradas pelo trabalho. Estes dados podem estar relacionados à necessidade de se manter no cargo e ao medo de perder o emprego, levando o indivíduo a trabalhar, mesmo na presença da dor. ${ }^{(17)}$

Uma pesquisa realizada por cirurgiões-dentista $(n=358)$ descreveu que estes profissionais estão entre os mais acometidos por DORT, causando afastamento temporário ou permanente da sua profissão. Dentre as regiões corporais mais acometidas, a coluna lombar (36 a 57\%) esteve em primeiro lugar, seguidados ombros (42\%) e da coluna cervical $(44 \%) .{ }^{(23)}$ Estes dado corroboram como presente estudo,onde houve um maior percentual de sintomas na coluna lombar $(63,3 \%)$ em técnicos de enfermagem, com alta prevalência de sintomas também em ombros e em região cervical. Similaridade também foi observado em outro estudo, ${ }^{(24)}$ onde profissionais fisioterapeutas relataram bastante dor lombar. Mesmo em profissões distintas, as dores foram frequentes na mesma região, provavelmente devido à desorganização postural e a falta de ergonomia.

Em segundo lugar, o atual estudo encontrou a dor nos tornozelos e pés $(56,7 \%)$, diferindo um pouco do trabalho anteriormente citado, talvez por conta da atividade realizada 
por cada profissional, já que os cirurgiões-dentista trabalham muito tempo em sedestração, diferentemente dos técnicos de enfermagem que passam a maior parte do tempo em ortostase. Em terceiro lugar, o local mais acometido foram os ombros $(51,7 \%)$, mostrando novamente uma similaridade com o trabalho realizado por Santos Filho e Barreto em 2001, ${ }^{(23)}$ justificando-se pelo fato das duas profissões utilizarem bastante os membros superiores durante a atividade laboral.

No trabalho realizado por Magnagoet al. em 2010, foi possível encontrar dados que corroboram com os resultados desta pesquisa, onde houve maior prevalência do sexo feminino (88\%) e maior queixa álgica na região lombar (71,5\%), o que demonstra a consequência do tipo de tarefa realizada por estes profissionais, podendo ter relação com o ritmo de trabalho, além da alta demanda e pouco tempo para realizá-las. ${ }^{(25,26)}$

Segundo a Consolidação das Leis Trabalhistas (CLT), ${ }^{(27)}$ a carga horária de trabalho não deve ser superior a 8 horas por dia, porém isto não é observado na maioria dos estudos. Nesta pesquisa os técnicos de enfermagem relataram trabalhar 9,5 $\pm 4,1$ horas/dia, algo próximo de um estudo realizado com costureiras profissionais que relataram $8,7 \pm 0,7$ horas/dia. ${ }^{(28)}$ Já em outro estudo, foi descrito que motoristas e cobradores de ônibus também atuavam com carga horaria superior ao que se foi orientado pela CLT. ${ }^{(29)}$ O trabalho dos técnicos de enfermagem pode ser extremamente desgastante devido aos movimentos realizados e a sobrecarga durante o manuseio dos pacientes (banho, mudança de decúbito, dentre outros), principalmente para quem atua em instituições hospitalares, sendo importante não ultrapassar os limites de carga horária nestes profissionais.

Existem cobranças relativas à prática de horários rígidos e ao trabalho por turno, com um pequeno período para descanso, ou até mesmo sem tempo para pausa, devido à alta demanda de trabalho. ${ }^{(28,30)}$ Desta forma inicia-se a interferência do trabalho na saúde do trabalhador, levando-se em conta as influências tanto biológicas como emocionais, devido às alterações dos ritmos circadianos, do ciclo sono-vigília e do sistema termorregulador. ${ }^{(18)}$ No presente estudo é possível observar a precocidade do aparecimento desses sintomas (4,9 anos), o que é um tempo relativamente pequeno quando comparado ao tempo de serviço que deve ser cumprido para se aposentar (30 anos para as mulheres e 35 anos para os homens).

Outro informação é que na comparação entre o tempo de atuação, aqueles com tempo de superior a 3 anos tiveram maiorfrequência de dor na região lombar $(p=0,02)$, o que pode ser justificado também pela maior faixa etária deste grupo $(38,5 \pm 9,5$ anos $)$ em relação a aqueles com tempo menor que 3 anos $(29,11 \pm 6,2$ anos). Não foi realizada a comparação por 
sexo, devido a quase maioria do estudo ser do sexo feminino, como também não foi feita a análise de diferentes grupos de IMC, já que a maioria encontrava-se na faixa de normalidade.

Dentre as limitações do estudo pode-se citar o tamanho amostral, o qual não foi maior devido a coleta ter sido realizada em apenas um hospital. Outra limitação do estudo foi não coletar o nível de atividade física destes indivíduos já que este pode ser um fator protetor para as alterações osteomusculares.

\section{CONCLUSÃO}

Cerca de mais da metade dos entrevistados referiram ter sentido dores nas regiões lombar, nos tornozelos e nos ombros, nos últimos 12 meses, com um tempo de trabalho relativamente pequeno (4,9 anos).O surgimento insidioso desses sintomas pode ser causado pelas posturas inadequadas durante a atividade laboral, pela alta demanda dos pacientes acamados, bem comopela alta carga horário de trabalho e ausência de pausas para descando.

Deve ser dada uma maior atenção às posturas realizadas por esses profissionais durante as atividades laborais, principalmente nas atividades que exijam força e agilidade, devido à sobrecarga durante o manuseio dos pacientes. A associação de medidas preventivas no trabalho com períodos adequados de descanso, posturas corretas durante o atendimento e ginástica laboral, podem influenciar na redução dessas dores e colaborar para a promoção da saúde dessa classe de trabalhadores.

\section{REFERÊNCIAS}

1. Carvalho AJFP, Alexandre NMC. Sintomas osteomusculares em professores do ensino fundamental. Revista bras.fisioter. 2006;10(1): 35-41.

2. Murofuse NT,Marziale MHP. Mudanças no trabalho e na vida de bancários portadores de lesões por esforços repetitivos: LER. Rev. latinoam. enferm. 2001;9(4): 19-25.

3. Picoloto D, Silveira E. Prevalência de sintomas osteomusculares e fatores associados em trabalhadores de uma indústria metalúrgica de Canoas - RS. Ciênc. saúde coletiva. 2008; 13(2):507-516.

4. Baptista PCP,MerighiMAB, Silva A. Angústia de mulheres trabalhadoras de enfermagem que adoecem por distúrbios osteomusculares relacionados ao trabalho. Rev. Bras. enferm. 2011;64(3): 438-444. 
5. Magnago TS, Lisboa MTL, Souza IEO, Moreira MC. Distúrbios musculo-esqueléticos em trabalhadores de enfermagem: associação com condições de trabalho. Rev. bras. enferm. 2007;60(6): 701-705.

6. Mergener RC,Kehrig TR,Traebert J. Sintomatologia músculo-esquelética relacionada ao Trabalho e Sua Relação com Qualidade de Vida in Bancários do Meio Oeste Catarinense. SocSaude. 2008;17(4): 171-181.

7. Mendes LF,Lancman S. Reabilitação de pacientes com LER/DORT: Contribuições da fisioterapia em grupo. Rev.bras. saúde ocup. 2010;35(121): 23-32.

8. Brasil. Instituto Nacional Do Seguro Social. Instrução Normativa INSS/DC No 98 , de 05 de Dezembro de 2003. Dispõe sobre atualização clínica das Lesões por Esforços Repetitivos (LER)/ Distúrbios Osteomusculares Relacionados ao Trabalho (DORT). Diario Oficial da União,2003.

9. Leite PC,Merighi MAB, Silva A. A vivência de uma trabalhadora de enfermagem portadora de lesão “De Quervain”. Rev. latinoam. enferm. 2007;15(2): 253-258.

10. Augusto VG, Sampaio RF, Tirado MGA, Mancini MC, Parreira VF. Um olhar sobre as LER/DORT no contexto clínico do fisioterapeuta. Rev.bras.fisioter. 2008;12(1): 49-56.

11. Barbosa MSA, Santos RM,Trezza MCSF. A vida do trabalhador antes e após a Lesão por Esforço Repetitivo (LER) e Doença Osteomuscular Relacionada ao Trabalho (DORT). Rev. bras. enfermagem. 2007;60(5): 491-496.

12. Tomasi E,Sant' anna GC,Oppelt AM,Petrini RM, Pereira IV,Sassi BT. Condições de trabalho e automedicação em profissionais da rede básica de saúde da zona urbana de Pelotas, RS. Rev. bras. epidemiol. 2007;10(1): 66-74.

13. Coluci, MZO, Alexandre NMC. Adaptação cultural de instrumento que avalia atividades do trabalho e sua relação com sintomas osteomusculares. Acta paulenferm. 2009;22(2): 149154.

14. Célia RCRS, Alexandre NMC. Aspectos ergonômicos e sintomas osteomusculares em um setor de transporte de pacientes. Rev. gaúch.enferm. 2004;25(1): 33-43.

15. Batiz EC, Vergara LGL,Licea OEA. Análise comparativa entre métodos de carregamento de cargas e análise postural de auxiliares de enfermagem. Produção.2012;22(2): 270-283.

16. Filho FL,Silva AAM, Lopes JMA, Lamy ZC, Simões VMF, Santos AM. Carga de trabalho de profissionais da saúde e eventos adversos durante ventilação mecânica em unidades de terapia intensiva neonatal. J. pediatr. 2011;87(6): 487-492.

17. Reis RJ, LaRoccaPF, Silveira AM,Bonilla IML,Goné AN, Martín M. Fatores relacionados ao absenteísmo por doença em profissionais de enfermagem. Rev. saúde pública. 2003; 37(5): 616-623. 
18. Gurgueira GP, Alexandre NMC, Filho HRC. Prevalência de Sintomas músculoesqueléticos em trabalhadoras de enfermagem. Rev. latinoam. enferm. 2003;11(5): 608-613.

19. Brasil, Ministério Da Saúde. Resolução 196/96 do Conselho Nacional de Saúde/MS Sobre Diretrizes e Normas Regulamentadoras de Pesquisa envolvendo seres humanos. Diário Oficial da União, 10 de outubro de 1996.

20. BarrosENC, Alexandre NMC. Cross-cultural adaptation of the Nordic musculoskeletal questionnaire. Int. Nurs. Rev. 2003;50(2):101-108.

21. Pinheiro FA,Troccoli BT, Carvalho CV. Validação do Questionário Nórdico de Sintomas Osteomusculares como medida de morbidade. Rev. saúde pública. 2002;36(3): 307-312.

22. Brandão AG, Horta BL, Tomasi E. Sintomas de Distúrbios osteomusculares em Bancários de Pelotas e Região: Prevalência e fatores associados. Rev. bras. epidemiol. 2005;8(3): 295305.

23. Filho SBS, Barreto SM. Atividade ocupacional e prevalência de dor osteomuscular em cirurgiões-dentistas de Belo Horizonte, Minas Gerais, Brasil: contribuição ao debate sobre os distúrbios osteomusculares relacionados ao trabalho. Cad. saúde pública. 2001;17(1):181-193.

24. Siqueira GR,Cahú FGM, Vieira RAG. Ocorrência de lombalgia em fisioterapeutas da cidade de Recife, Pernambuco. Rev. bras. fisioter. 2008;12(3): 222-227.

25. Magnago TSBS, Lisboa MTL,Griep RH,Kirchhof ALC,Camponogara CQN et al. Condições de trabalho, características sociodemográficas e distúrbios musculoesqueléticos em trabalhadores de enfermagem. Acta paul. enferm. 2010;23(2): 187-193.

26. Mello MC,Fugulin FMT,Gaidzinsk RR. O tempo no processo de trabalho em saúde: uma abordagem sociológica. Acta paul.enferm. 2007;20(1): 87-90.

27. Brasil. Ministério do Trabalho e Emprego. Consolidação das leis do trabalho. Decreto-Lei $n^{\circ} 5452$ de $1^{\circ}$ de maio de 1943. Brasília (DF): LTr; 2007.

28. Freitas FCT,Barbosa LH,Alves LA,Marziale MHP,Robazzi MLCC. Avaliação Cinesiológica e Sintomatológica de Membros Inferiores de Costureiros Industriais. Rev. enferm. 2009;17(2):170-175.

29. Carneiro LRV,Coqueiro RS,Freire MO,Barbosa AR. Sintomas de distúrbios osteomusculares em motoristas e cobradores de ônibus. Rev. bras. cineantropom. desempenho hum. 2007; 9(3): 277-283.

30. Murofuse NT,Marziale MHP. Doenças do Sistema osteomuscular em Trabalhadores de Enfermagem. Rev. latinoam. enferm. 2005;13(3): 364-373. 